Radiologe 2021 · 61:251-257

https://doi.org/10.1007/s00117-021-00816-4

Angenommen: 19. Januar 2021

Online publiziert: 11. Februar 2021

(c) Springer Medizin Verlag GmbH, ein Teil von Springer Nature 2021

Bei der Entdeckung von entzündlichen Herden im Myelon durchläuft ein Neuroradiologe häufig zwei Phasen. Die erste Phase ist geprägt durch die Erleichterung, dass eine Ursache für die Symptome gefunden wurde. Die zweite Phase ist schon etwas unangenehmer, insbesondere wenn ein Neurologe daneben sitzt und mit hoffnungsvollen Augen fragt: „Und, was hat der Patient?"

An dieser Stelle gibt es zwei Strategien: Die einfachere ist, die Läsionen zu beschreiben und den Neurologen seinem Schicksal zu überlassen, sprich, er muss selbst herausfinden, um welche Krankheit es sich handelt. Die zweite und schwierigere Strategie ist, anhand der Morphologie die Differenzialdiagnosen möglichst weit einzuschränken. Dieser Beitrag ist vorwiegend für Anhänger der letzteren Strategie interessant, wobei der Fokus auf entzündliche Veränderungen gelegt wird.

Ferner folgt diese Übersicht der liebgewordenen Tradition der Kliniker, den Neuroradiologen mit möglichst wenig (wenn überhaupt) Informationen bezüglich Symptomatik und Anamnese zu versorgen, das heißt, es wird nur am Rande auf die Korrelation zwischen neurologischen Untersuchungsergebnissen und Laborwerten sowie Bildmorphologie eingegangen.

Die wichtigsten demyelinisierende Erkrankungen sind:

- Multiple Sklerose (MS)

- Akute disseminierte Enzephalomyelitis (ADEM)

\author{
A. Bachhuber \\ Homburg, Deutschland
}

\title{
Entzündliche Rückenmarkerkrankungen und Querschnittsmyelitis
}

- Neuromyelitis-optica-SpektrumErkrankungen (NMOSD)

- Idiopathische transverse Myelitis (ITM)

Ferner treten auch bei anderen Autoimmunerkrankungen und Infektionen Myelopathien auf:

- Systemischer Lupus erythematodes (SLE)

- Sjögren-Syndrom (SS)

- Antiphospholipid-Syndrom (APS)

- Sarkoidose

- (Neuro-) Morbus Behçet (NBD)

- Paraneoplastische Myelitis

- Infektiöse Myelitis

Die Aufzählung erhebt keinen Anspruch auf Vollständigkeit. Am Schluss wird noch kurz auf den $\mathrm{B}_{12^{-}}$und Kupfermangel eingegangen, da es sich hierbei ebenfalls um eine wichtige Differenzialdiagnose handelt.

Die wichtigsten Punkte zu den jeweiligen Erkrankungen sind in 0 Tab. 1 zusammengefasst.

\section{Multiple Sklerose}

Die MS ist in unseren Breitengraden die häufigste demyelinisierende Erkrankung. Beietwa 90 \% der Patienten ist das Myelon mitbetroffen, und bei etwa $20 \%$ alleiniger Manifestationsort [8]. Die Läsionen bevorzugen das Zervikalmark und sind unter 2 Segmente lang. Meist sind sie peripher dorsolateral gelegen mit einer ovoiden Form ( $\bullet \mathbf{A b b} . \mathbf{1 a}, \mathbf{b})$. Die graue Substanz ist deutlicher betroffen als im Gehirn. Typischerweise sind weniger als $50 \%$ des Myelonquerschnitts entzündlich verändert, und akute Läsionen sind im allgemeinen kontrastmittelaffin, wobei es gelegentlich zu einer randständigen Kontrastmittelaufnahme und einer Restriktion in der diffusionsgewichteten Bildgebung (DWI) kommen kann. Dabei korreliert nicht die Anzahl der Läsionen, sondern das Ausmaß der spinalen Atrophie mit der körperlichen Behinderung. Bei der progressiven MS kann diese sogar als prädiktiver Marker für den Verlauf der Erkrankung dienen.

\section{Akute disseminierte Enzephalomyelitis}

Während die MS sich durch einen schubförmig oder chronischen progredienten Verlauf auszeichnet, ist die akute disseminierte Enzephalomyelitis (wie der Name schon sagt) akut und monophasisch. Typischerweise tritt sie innerhalb von 4 Wochen nach viralen Infekten oder Impfungen auf. Häufig fehlen die oligoklonalen Baden im Liquor [14]. Im Gegensatz zur MS befinden sich die Läsionen bevorzugt thorakal und sind länger als 2 Wirbelkörper, obwohl sie weniger als die Hälfte des Myelonquerschnitts betreffen. Unterschiedlich ist auch, dass sie aufgrund des perifokalen Ödems nicht so scharf abgrenzbar sind. Nicht alle Veränderungen sind kontrastmittelaffin, was nahelegt, dass die Erkrankung einen schubförmigen subklinischen Verlauf hat. Um die akute disseminierte Enzephalomyelitis eindeutig $\mathrm{zu}$ diagnostizieren, sind zusätzlich große konfluierende gliotische Veränderungen im Gehirn notwendig. 
Tab. 1 Kurze Orientierungshilfe der Myelitiden und ihren morphologischen Charakteristika

\begin{tabular}{|c|c|c|c|c|c|c|c|c|c|c|}
\hline \multirow[t]{2}{*}{ Erkrankung } & \multicolumn{2}{|c|}{$\begin{array}{l}\text { Länge der Läsion } \\
\text { (sagittal) }\end{array}$} & \multicolumn{2}{|c|}{$\begin{array}{l}\text { Bevorzugte Loka- } \\
\text { lisation (sagittal) }\end{array}$} & \multicolumn{2}{|c|}{$\begin{array}{l}\text { Bevorzugte Lo- } \\
\text { kalisation (axial) }\end{array}$} & \multicolumn{2}{|c|}{$\begin{array}{l}\text { Ausmaß Mye- } \\
\text { lonquerschnitt }\end{array}$} & \multirow[t]{2}{*}{$\begin{array}{l}\text { KM-Af- } \\
\text { finität }\end{array}$} & \multirow[t]{2}{*}{ Besonderheit } \\
\hline & $\begin{array}{l}<3 \\
\text { Wirbel- } \\
\text { körper }\end{array}$ & $\begin{array}{l}\geq 3 \\
\text { Wirbel- } \\
\text { körper }\end{array}$ & Zervikal & Thorakal & Zentral & Peripher & $<50 \%$ & $>50 \%$ & & \\
\hline $\begin{array}{l}\text { Multiple Sklero- } \\
\text { se }\end{array}$ & + & $\sim$ & + & $\sim$ & $\sim$ & + & + & $\sim$ & $+/-$ & $\begin{array}{l}\text { Multifokale Läsionen } \\
\text { meist dorsolateral, ovoid }\end{array}$ \\
\hline $\begin{array}{l}\text { Akute disse- } \\
\text { minierte Enze- } \\
\text { phalomyelitis }\end{array}$ & $\sim$ & + & $\sim$ & $\sim$ & $\sim$ & $\sim$ & + & $\sim$ & $+/-$ & $\begin{array}{l}\text { Unscharfe Abgrenzbarkeit wegen perifo- } \\
\text { kalem Ödem }\end{array}$ \\
\hline $\begin{array}{l}\text { Neuromyelitis- } \\
\text { optica-Spek- } \\
\text { trum-Erkran- } \\
\text { kungen }\end{array}$ & $\sim$ & + & + & $\sim$ & + & $\sim$ & $\sim$ & $\sim$ & + & $\begin{array}{l}\text { Unifokale Läsionen } \\
\text { T1w-Hypointensitäten als Zeichen der } \\
\text { Nekrosen }\end{array}$ \\
\hline $\begin{array}{l}\text { NMOSD mit } \\
\text { MOG-Antikör- } \\
\text { pern }\end{array}$ & + & + & $\sim$ & + & + & $\sim$ & $\sim$ & $\sim$ & + & $\begin{array}{l}\text { Weniger Nekrosen, KM-Affinität entlang } \\
\text { des Zentralkanals }\end{array}$ \\
\hline $\begin{array}{l}\text { Idiopathische } \\
\text { Transverse } \\
\text { Myelitis }\end{array}$ & $\sim$ & + & + & + & + & $\sim$ & $\sim$ & + & $+/-$ & \\
\hline $\begin{array}{l}\text { Systemischer } \\
\text { Lupus erythe- } \\
\text { matodes }\end{array}$ & $\sim$ & + & + & + & + & $\sim$ & $\sim$ & $\sim$ & + & \\
\hline $\begin{array}{l}\text { Sjögren-Syn- } \\
\text { drom }\end{array}$ & $\sim$ & + & + & $\sim$ & + & $\sim$ & $\sim$ & $\sim$ & $\sim$ & Auch Optikusneuritis \\
\hline $\begin{array}{l}\text { Antiphospho- } \\
\text { lipid-Syndrom }\end{array}$ & $\sim$ & + & $\sim$ & + & $\sim$ & $\sim$ & $\sim$ & $\sim$ & $\sim$ & Unregelmäßige T2w-Hyperintensität \\
\hline Sarkoidose & $\sim$ & + & + & $\sim$ & $\sim$ & + & $\sim$ & $\sim$ & $\sim$ & $\begin{array}{l}\text { KM-Affinität persistiert über } 2 \text { Monate, } \\
\text { subpiale KM-Aufnahme im dorsalen } \\
\text { Myelon } \rightarrow \text { „trident sign“ }\end{array}$ \\
\hline $\begin{array}{l}\text { (Neuro-) Mor- } \\
\text { bus Behçet }\end{array}$ & $\sim$ & + & + & + & + & $\sim$ & $\sim$ & + & $\sim$ & $\begin{array}{l}\text { Kleine Hämorrhagien, Bagel-Sign ( } \mathrm{T} 2 \mathrm{w} \\
\text { zentral hypointens, peripher hyperintens) } \\
\text { Motor-Neuron-Muster } \rightarrow \text { Signalsteigerun } \\
\text { am Cornu anterius }\end{array}$ \\
\hline $\begin{array}{l}\text { Paraneoplas- } \\
\text { tische Myelitis }\end{array}$ & $\sim$ & + & $\sim$ & + & + & $\sim$ & $\sim$ & $\sim$ & + & $\begin{array}{l}\text { Owl's Eye Zeichen, ähnlich spinaler } \\
\text { Ischämie }\end{array}$ \\
\hline $\begin{array}{l}\text { Myelitis bei } \\
\text { COVID-19 }\end{array}$ & + & + & $\sim$ & $\sim$ & $\sim$ & $\sim$ & $\sim$ & $\sim$ & - & \\
\hline $\begin{array}{l}\mathrm{B}_{12} \text { - und Kupfer- } \\
\text { mangel }\end{array}$ & $\sim$ & $\sim$ & + & + & $\sim$ & + & $\sim$ & $\sim$ & - & Symmetrisch Columna dorsalis \\
\hline
\end{tabular}

\section{Neuromyelitis-optica- Spektrum-Erkrankungen}

Während die MS ihren Inzidenzhöhenpunkt um das 29. Lebensjahr hat, ist dieser bei der Neuromyelitis-optica-Spektrum-Erkrankung etwa 10 Jahre später. Es handelt sich um eine monophasische oder schubförmige demyelinisierende entzündliche Erkrankung des ZNS, wobei vorwiegend die Astrozyten betroffen sind. Früher ging man davon aus, dass die Läsionen nur im Myelon und in den Nervi optici, zu finden sind. Durch verbesserte Diagnostik konnten inzwischen auch weitere zerebrale Läsionen nachgewiesen werden. Typischerweise finden sich im Serum Aquaporin-4-Antikörper (AQP4-Ak). Deren Antigene sind zusätzlich auch am Hypothalamus und an der periependymalen Region lokalisiert, v.a. um das Aquädukt und am 3. und 4. Ventrikel [11], weswegen häufig eine Verwechslung mit der MS auftritt.
Dass es sich hier nicht um eine Rose handelt, der man einen anderen Namen gibt, zeigt sich am Versagen der typischen MS-Therapeutika, welche teilweise den Krankheitsverlauf verschlimmern können [4]. Der Druck auf den Neuroradiologen ist hier umso höher, da ein Teil der Patienten negativ für AQP4-A ist.

Meist sind die Demyelinisierungsherde länger als 2 Wirbelkörper und im Gegensatz zur MS unifokal. Interessan- 
Hier steht eine Anzeige.

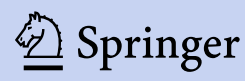


terweise korreliert der Aquaporin-4-Antikörper-Spiegel mit der Länge der Läsionen [12]. Die bevorzugte Lokalisation ist zervikal, teilweise reichen sie bis in das thorakale Myelon und in die Medulla oblongata. Ähnlich der multiplen Sklerose kann es zu einer (ringförmigen) Kontrastmittelaufnahme und fokaler Schwellung kommen. Da vorwiegend die graue Substanz betroffen, ist die Lokalisation zentral und meist symmetrisch (• Abb. 2a, b). Wenn sich in der T1w-Hypointensitäten in den Herden zeigen, reflektiert dies den größeren Gewebeschaden, und ist typisch für die NMOSD.

Eine Untergruppe der AQP4-Ak-negativen Patienten hat Antikörper gegen das Myelin Oligodendrozyten Glykoprotein (MOG). Diese Variante ist prognostisch günstiger und unterscheidet sich bildmorphologisch von den anderen NMOSD-Fällen. Etwa $44 \%$ haben kurze, meist thorakolumbale Läsionen. Zwar ist auch hier die graue Substanz bevorzugt betroffen, jedoch kommt es selten zu Nekrosen und Atrophie. Die Kontrastmittelaufnahme wird als dünn und entlang des Zentralkanals beschrieben. Da etwa $25 \%$ der Patienten zusätzlich eine maligne Grunderkrankung haben, ist ein Tumorscreening obligat.

\section{Idiopathische transverse Myelitis}

Die idiopathische transverse Myelitis ist eine Ausschlussdiagnose und wird umso seltener, je weiter die diagnostischen Möglichkeiten voranschreiten. Da es sich um ein Konglomerat aus verschiedenen Entitäten handelt, ist es nicht verwunderlich, dass es keine einheitliche Morphologie gibt. Die Größe der zentral gelegenen Herde ist häufig mehr als $50 \%$ des Myelonquerschnitts. Sowohl das zervikale als auch das thorakale Myelon sind betroffen. Die Länge beträgt etwa 3-4 Segmente. Eine Schwellung des Rückenmarks wird beschrieben, ebenso variable Kontrastmittelaffinität.

\section{Systemischer Lupus erythematodes}

Systemischer Lupus erythematodes ist eine systemische Autoimmunerkran-

Radiologe 2021 · 61:251-257 https://doi.org/10.1007/s00117-021-00816-4

(c) Springer Medizin Verlag GmbH, ein Teil von Springer Nature 2021

\section{A. Bachhuber \\ Entzündliche Rückenmarkerkrankungen und Querschnittsmyelitis}

Zusammenfassung

Hintergrund. Entzündliche Rückenmarkerkrankungen zeigen MR-morphologisch recht einheitliche Bilder, dennoch sind deren richtige Zuordnung zu einer Krankheit für die Therapie und weiteren Verlauf entscheidend. Fragestellung. Welche Merkmale der Läsionen erlauben eine richtige Einordnung? Material und Methode. Recherche von Artikeln in pubmed.gov, die Myelitiden verschiedener Ätiologie beschreiben.

Ergebnisse. Die Länge der Läsionen, deren Lokalisation im Rückenmark sowohl bezüglich Höhe als auch im Myelonquerschnitt, und das Kontrastmittelverhalten erlauben in vielen Fällen zumindest eine Eingrenzung der Differenzialdiagnosen. Zum Überblick werden die wichtigsten Charakteristika in einer Tabelle zusammengefasst.

Schlussfolgerung. Eine sichere Zuordnung ist nur in Ausnahmefällen möglich, da sich viele Entitäten überlappen. Besonders schwierig ist dies bei Erkrankungen, die seltener vorkommen, und deswegen diesbezüglich wenig Erfahrung vorhanden ist.

Schlüsselwörter

Multiple Sklerose - Akute disseminierte Enzephalomyelitis - Neuromyelitis-opticaSpektrum-Erkrankungen · Idiopathische transverse Myelitis · Myelitiden

\section{Inflammatory spinal cord diseases and transverse myelitis}

\section{Abstract}

Background. Inflammatory spinal cord diseases are difficult to differentiate based on magnetic resonance (MR) morphological properties. However, correct diagnosis is crucial for treatment and outcome.

Objectives. What MR characteristics allow correct classification?

Materials and methods. A literature search for articles published in PubMed about various forms of transverse myelitis was performed.

Results. The length of the lesions, the level and location in the cross-section, and the enhancement pattern gives good clues for the correct diagnosis. The most important characteristics have been summarized in a table.

Conclusions. In most cases, it is not possible to make a conclusive diagnosis, because many diseases overlap.

\section{Keywords}

Multiple sclerosis - Acute disseminated encephalomyelitis - Neuromyelitis optica spectrum disorders $\cdot$ Idiopathic transverse myelitis - Myelitis kung und betrifft in etwa $60 \%$ der Fälle das ZNS. Eine Myelitis kommt bei 1-2\% der Patienten vor [5]. Der vermutete Pathomechanismus ist die Kombination aus Vaskulitis und arteriellen Thromben. Auch bei klinischem Hinweis auf eine Myelitis zeigen etwa 30\% aller Patienten keine Auffälligkeiten in der MRT, weswegen dieses etwa 2-7 Tagen später wiederholt werden sollte. In mehreren Veröffentlichungen wird eine Koexistenz von SLE und NMOSD beschrieben, was auf eine intrinsische Hyperaktivität der B-Zellen hinweist. Dies macht die Diagnose sowohl klinisch als auch bildmorphologisch deutlich schwieriger, da sich beide Erkrankungen überlappen. Es wird klinisch unterschieden zwischen
Gray-matter-Myelitis (Paresen und Hyporeflexie) und einer White-matterMyelitis (Spastizität und Hyperreflexie). Die Unterscheidung ist nicht nur akademischer Natur, es konnte nachgewiesen werden, dass AQP4-Ak vor allem bei der White-matter-Myelitis vorhanden sind.

Die Läsionen sind bei 71,4\% der Patienten länger als 3 Wirbelkörper [6]. Wie bei der NMOSD kommt es zu einer Myelonschwellung aufgrund des fokalen Ödems, einen bevorzugten Befall des zentralen Myelons sowie Kontrastmittelaufnahme. Die Herde können sich bis in die Medulla oblongata und sogar über das gesamte Mark ausbreiten [2]. 

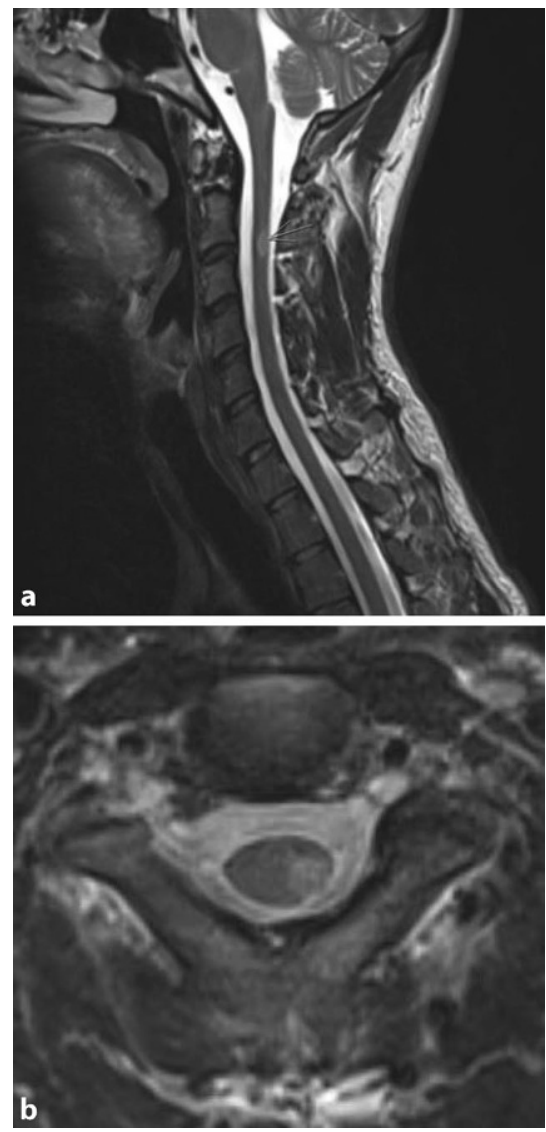

Abb. $1 \Delta$ a Magnetresonanztomographie (MRT) T2w: Kurzstreckiger MS-Herd im dorsalen zervikalen Myelon. $\mathbf{b}$ T2w: In den axialen Schichten zeigte sich die periphere Lage des MS-Herdes mit der ovoiden Konfiguration

\section{Sjögren-Syndrom}

Auch wenn die Beteiligung des zentralen Nervensystems mit einer Prävalenz von $4,8 \%$ selten ist [9], können neurologische Ausfälle der Diagnose des Sjögren-Syndroms vorausgehen. Die Patienten werden meist zuerst mit MS und erst nach Manifestation des typischen Phänotyps mit Sjögren-Syndrom diagnostiziert. Die Demyelinisierungsherde sind vorwiegend zervikal und zentral mit einer Länge von 3-5 Wirbelkörper. Da mehrere Patienten auch eine Optikusneuritis, zerebrale Läsionen und AQP4$\mathrm{Ak}$ aufweisen, erfüllen sie teilweise die Diagnosekriterien für eine koexistierende MS oder NMOSD.
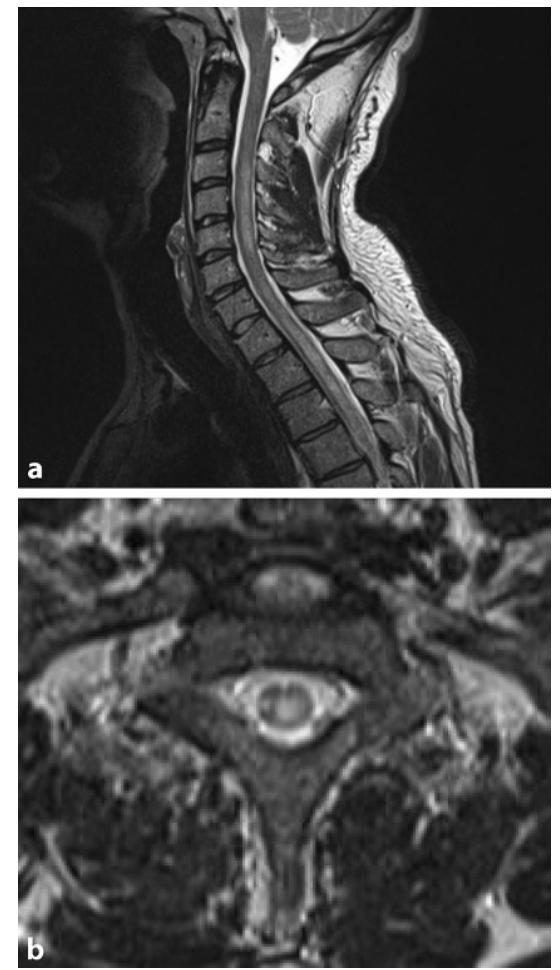

Abb. $2 \Delta$ a Magnetresonanztomographie (MRT) T2w: langstreckiger Herd einer Neuromyelitis-optica-Spektrum-Erkrankung (NMOSD), der sowohl das zervikale als auch das thorakale Myelon betrifft. b T2w: zentrale Lage des NMOSD-Herds im Myelon

\section{Antiphospholipid-Syndrom}

Die Myelitis ist mit weniger als $1 \%$ eine seltene Komplikation des Antiphospholipid-Syndroms [1]. Als Pathomechanismus wird eine Vaskulitis aufgrund von Immunkomplexen vermutet. Eine andere These ist, dass die Antikörper direkt mit Phospholipiden des Myelons interagieren. Der Umstand, dass ein Teil der Patienten positiv für AQP4-Ak sind, erschwert auch dem Kliniker die Abgrenzung zu anderen Erkrankungen.

Die Läsionen befinden sich meist auf thorakaler Höhe und zeigt unregelmäßige T2-Hyperintensität mit Myelonschwellung. Auch diese sind meist 3 oder mehr Segmente lang.
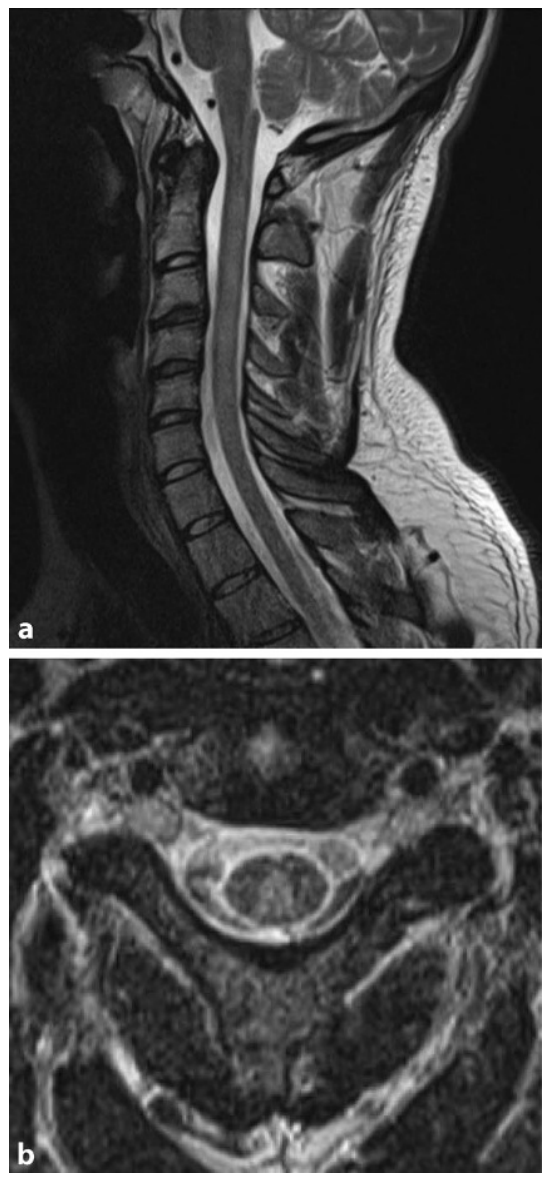

Abb. $3 \Delta$ a Magnetresonanztomographie (MRT) T2w: zervikaler, langstreckiger Herd bei $B_{12}$-Mangel. b T2w: Hyperintensität der Columna dorsalis in den axialen Schichten

\section{Sarkoidose}

Die Sarkoidose ist eine Multisystemerkrankung mit nichtverkäsenden Granulomen, die primär die Lunge mit den intrathorakalen Lymphknoten affektiert. Bei 5-15\% der Patienten kommt es zu einem ZNS-Befall, bei weniger als $1 \% \mathrm{zu}$ einer Myelitis. Auch hier ist es schwierig, zwischen spinaler Sarkoidose (SCS) und NMOSD zu unterscheiden. Beide haben eine Bevorzugung des zervikalen Rückenmarks, auch wenn diese Präferenz für die SCS nicht so deutlich ist. Ebenfalls sind die Herde häufig 3 oder mehr Segmente lang. Zwei Punkte treten jedoch nur bei der Sarkoidose und nicht bei der NMOSD auf:

1. persistierende Kontrastmittelaffinität der Demyelinisierungsherde über 2 Monate, 
2. subpiale Kontrastmittelaufnahme im dorsalen Myelon (axiale Schichten), was von manchen Autoren als ,trident sign" bezeichnet wird (ähnlich dem Zeichen bei der osmotische Demyelinisierung; [3]).

\section{(Neuro-) Morbus Behçet}

Beim (Neuro-) Morbus Behçet handelt es sich um eine Multisystemvaskulitis, welche meist mit schmerzhaften mukokutanen Läsionen beginnt. Die höchste Prävalenz ist im mittleren Osten und im Pazifikraum. Eine Beteiligung des ZNS erfolgt bei etwa 9,4\% aller Patienten, hiervon leiden zwischen 2,5-30\% an Myelitiden und gelten als prognostisch ungünstig.

Die meisten haben Myelonläsionen von 3 und mehr Segmenten (teilweise auch entlang des gesamten Rückenmarks) und ähneln somit der NMSOD. Die Lokalisation wird als zervikal und thorakal beschrieben. Wiederum ist die zentrale Region des Myelons vorwiegend betroffen und umfasst meist mehr als die Hälfte des Querschnitts. Da postkapillare Venolen involviert sind, können in der suszeptibilitätsgewichteten Bildgebung kleine Hämorrhagien nachgewiesen werden. Es käme wieder zu einem diagnostischen Dilemma, gäbe es nicht 2 Zeichen, die typisch für NBD sind:

3. das Bagel-Sign,

4. das Motor-Neuron-Muster.

Das Bagel-Sign wird als Läsion mit zentraler Hypointensität in der T2w und hyperintensem Ring beschrieben, wobei das Kontrastmittelverhalten variabel ist. Es ist besonders prominent bei langen Läsionen. Das Motor-Neuron-Muster bezeichnet in der T2w eine Signalsteigerung des Cornu anterius medullae spinalis [13].

\section{Paraneoplastische Myelitis}

Die Pathogenese der paraneoplastischen Myelitis ist vermutlich eine autoimmune Reaktion gegen gemeinsamen Antigenen des Tumors und des Nervensystems. Bei der Enzephalomyelitis ist der häufigste Antikörper anti-Hu, welcher bei Lungen- und Mammakarzinomen vorkommt. Ein weiterer Antikörper richtet sich gegen das Collapsin Response Mediator Protein 5 (CRMP5) und führt $\mathrm{zu}$ einer Retinitis und Optikusneuritis sowie in $15 \%$ der Fälle zu einer Myelitis. Nicht in jedem Fall können Antikörper gefunden werden. Die körperliche Behinderung entwickelt sich schnell und bessert sich nur unzureichend nach Behandlung.

Die Herde betreffen vorwiegend die graue Substanz und sind symmetrisch, wodurch sie ein Owl's Eye-Zeichen oder Snake's Eye-Zeichen wie bei der spinalen Ischämie vortäuschen können. Die Kontrastmittelaufnahme ist gleichmäßig und nicht fleckig. Die Länge beträgt 3 Segmente oder mehr.

\section{Infektiöse Myelitis}

Die infektiöse Myelitis tritt bevorzugt bei Drogenabhängigen, nach Operationen und eingeschränkter Immunabwehr auf. Die bildgebende Diagnostik zeigt eine diffuse T2w-Hyperintensität mit Myelonödem sowie möglichen DWI-Restriktionen. Insgesamt ist das Bild unspezifisch, weswegen weitere klinische Angaben und Liquordiagnostik notwendig sind. Selbst die ringförmige Kontrastmittelaffinität bei Abszessen findet sich gelegentlich bei autoimmunen Prozessen [10].

Im Zeitalter der COVID-19-Pandemie darf natürlich nicht die Erwähnung der Corona-assoziierten Myelitiden fehlen. Seit dem Ausbruch in der chinesischen Stadt Wuhan im Dezember 2019 hat sich das Virus schnell über die Welt verbreitet und ist inzwischen fast in aller Munde. Es bindet an den Angiotensin-konvertierendes Enzym 2(ACE2)Rezeptor, welcher sich nicht nur auf dem respiratorischen Epithel, sondern auch auf den Gliazellen des Gehirns und des Rückenmarks befindet [7]. Wahrscheinlich erreicht es über die Lamina cribrosa den Bulbus olfactorius und damit das zentrale Nervensystem. Neben dem Virus selbst verursacht das Immunsystem durch einen Zytokinsturm (v. a. Interleukin 6) weiteren Endothelund Gliazellenschaden, der eine Ausbreitung und zusätzliche Schädigung begünstigt. Bisher wurden nur wenige
Myelitiden mit dem COVID-19-Virus in Zusammenhang gebracht. Die spinale Symptomatik sind sensomotorische Ausfälle bis hin zur Plegie der Extremitäten sowie autonome Störungen mit Harnund Blaseninkontinenz. In der T2w werden Signalsteigerungen über die Länge von 2-4 Wirbelkörpern ohne signifikante Kontrastmittelaufnahme beschrieben. Eine bildmorphologische zerebrale Mitbeteiligung ist nicht obligat. Aufgrund der noch geringen Fallzahl konnte bisher kein spezifisches Befallsmuster gefunden werden.

\section{$B_{12}$ - und Kupfermangel}

Ein $\mathrm{B}_{12^{-}}$und Kupfermangel präsentiert sich als subakute progressive Myelopathie. Sie manifestiert sich bildmorphologisch in der Columna dorsalis des Rückenmarks mit einer bilateralen symmetrischen Signalsteigerung in der T2w, die keine Kontrastmittelaffinität aufweist (- Abb. 3). Primär ist das Zervikal- und Thorakalmark betroffen.

\section{Fazit für die Praxis}

- Trotz unterschiedlicher Ätiologie zeigen Myelitiden in der Magnetresonanztomographie teilweise sehr ähnliche Erscheinungsbilder.

- Das Hauptproblem besteht darin, dass es einen fließenden Übergang zwischen den einzelnen Entitäten gibt, wie z. B. an der Komorbidität der Neuromyelitis-optica-SpektrumErkrankungen (NMOSD) mit weiteren Autoimmunerkrankungen zu sehen ist.

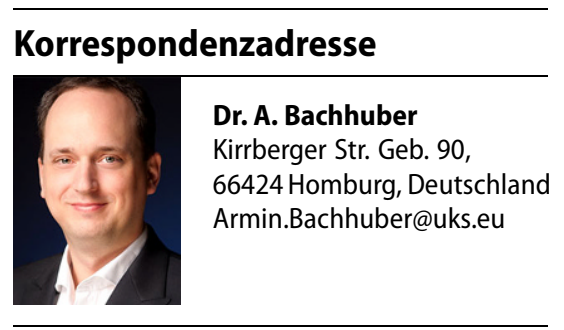

Einhaltung ethischer Richtlinien

Interessenkonflikt. A. Bachhuber gibt an, dass kein Interessenkonflikt besteht. 
Für diesen Beitrag wurden von den Autoren keine Studien an Menschen oder Tieren durchgeführt. Für die aufgeführten Studien gelten die jeweils dort angegebenen ethischen Richtlinien.

\section{Literatur}

1. Cervera R, Piette JC, Font J et al (2002) Antiphospholipid syndrome: clinical and immunologic manifestations and patterns of disease expression in a cohort of 1,000 patients. Arthritis Rheum 46:1019-1027

2. Deodhar AA, Hochenedel T, Bennett RM (1999) Longitudinal involvement of the spinal cord in a patient with lupus related transverse myelitis. JRheumatol 26:446-449

3. Flanagan EP, Kaufmann TJ, Krecke KN et al (2016) Discriminating long myelitis of neuromyelitis optica from sarcoidosis. Ann Neurol 79:437-447

4. Jarius S, Wildemann B, Paul F (2014) Neuromyelitis optica: clinical features, immunopathogenesis and treatment. Clin Exp Immunol 176:149-164

5. Kovacs B, Lafferty TL, Brent LH et al (2000) Transverse myelopathy in systemic lupus erythematosus: an analysis of 14 cases and review of the literature. Ann Rheum Dis 59:120-124

6. LiXY, Xiao P, Xiao HB et al (2014) Myelitis in systemic lupus erythematosus frequently manifests as longitudinal and sometimes occurs at low disease activity. Lupus 23:1178-1186

7. LiYC,BaiWZ,HashikawaT(2020)Theneuroinvasive potential of SARS-CoV2 may play a role in the respiratory failure of COVID-19 patients. J Med Virol 92:552-555

8. Lycklama G, Thompson A, Filippi M et al (2003) Spinal-cord MRI in multiple sclerosis. Lancet Neurol 2:555-562

9. Massara A, Bonazza S, Castellino G et al (2010) Central nervous system involvement in Sjogren's syndrome: unusual, but not unremarkable-clinical, serological characteristics and outcomes in a large cohort of Italian patients. Rheumatology (Oxford) 49:1540-1549

10. MurphyKJ, Brunberg JA, QuintDJetal (1998)Spinal cord infection: myelitis and abscess formation. AJNR Am J Neuroradiol 19:341-348

11. Pittock SJ, Weinshenker BG, Lucchinetti CF et al (2006) Neuromyelitis optica brain lesions localized at sites of high aquaporin 4 expression. Arch Neuro 63:964-968

12. Takahashi T, Fujihara K, Nakashima I et al (2007) Anti-aquaporin-4 antibody is involved in the pathogenesis of NMO: a study on antibody titre. Brain 130:1235-1243

13. Uygunoglu U, Siva A (2018) Behcet's syndrome and nervous system involvement. Curr Neurol Neurosci Rep 18:35

14. Young NP, Weinshenker BG, Lucchinetti CF (2008) Acute disseminated encephalomyelitis: current understanding and controversies. Semin Neurol 28:84-94

\section{Diastolische Herzschwäche: Diagnostik mit Echtzeit-MRT kann Alternative zur Herzkatheter-Untersuchung sein}

Bei einer diastolischen Herzschwäche, die auch Heart failure with preserved ejecton fraction (HFpEF) genannt wird, bleibt die Pumpkraft des Herzens erhalten, aber die linke Herzkammer ist steif und füllt sich nicht ausreichend mit sauerstoffreichem Blut. Die Patienten leiden unter Atemnot, Wassereinlagerungen und sind körperlich zunehmend weniger leistungsfähig. Mit nichtinvasiven Methoden, wie einer Ultraschalluntersuchung, war die HFpEF bisher schwer zu diagnostizieren und wurde oft erst spät erkannt.

In der Studie HFpEF-stress-DZHK17 konnten die Wissenschaftler um Studienleiter Professor Dr. Andreas Schuster, Klinik für Kardiologie und Pneumologie der Universitätsmedizin Göttingen (UMG), nun zeigen, dass die diastolische Herzschwäche mithilfe einer neuen nicht-invasiven Echtzeit-MRTTechnologie präzise diagnostiziert und somit eine Herzkatheter-Untersuchung zukünftig möglicherweise vermieden werden kann. Professor Dr. Martin Uecker, Institut für Diagnostische und Interventionelle Radiologie der UMG, sowie Dr. Shuo Zhang und Professor Dr. Jens Frahm vom Max-Planck-Institut für biophysikalische Chemie in Göttingen entwickelten die neue MRT-Technologie. Sie erlaubt es, MRT-Messungen am Herzen unter Belastung durchzuführen. Denn die Patienten können bei der MRT-Untersuchung weiteratmen und müssen nicht, wie bisher, währenddessen den Atem anhalten.

\section{Eingeschränkte Herzfunktion wird un- ter Belastung deutlich}

Bei der am Göttingen Campus entwickelten Methode ist auf der Untersuchungsliege eine Art Hometrainer installiert. Die Besonderheit liegt in den nicht-magnetischen Bauteilen des MRT Ergometers, die dessen Einsatz im Magnetfeld des Magnetresonanztomographen ermöglichen. Die Patienten fahren liegend Fahrrad, dabei vermisst das MRTGerät ihr Herz. Die Bilder können die Ärzte während der Untersuchung auf einem Bildschirm verfolgen und so genau beurteilen, wie gut das Pumporgan des Patienten funktioniert. „Wir sehen im MRT, wie das Herz schlägt, wie es sich füllt und wieder entleert", erklärt der Erstautor der Studie, Privatdozent Dr. Sören Backhaus, Klinik für Kardiologie und Pneumologie der UMG. „Mit dem MRT können wir daher direkt die krankmachende Veränderung am Herzen messen und nicht nur die Konsequenzen beurteilen." Bei Patienten mit HFpEF ist dabei zu sehen, dass die Leistung des linken Vorhofs des Herzens eingeschränkt ist. Das ist die erste Herzkammer, die das mit Sauerstoff beladene Blut nach der Lunge erreicht. „Diese eingeschränkte Funktion wird aber nur deutlich, wenn die Patienten sich bewegen und damit Herz und Kreislauf belasten", so Backhaus.

\section{Multizentrische Studien geplant}

Bei dem bisherigen Goldstandard der Herzuntersuchung wird ein Katheter über die Lungenarterie in das Herz geschoben und misst die Veränderung des Lungendrucks, während sich die Patienten bewegen. Bei Patienten mit einer diastolischen Herzschwäche staut sich unter Belastung das Blut in die Lunge zurück, sodass der Lungendruck zunimmt. Die Untersuchung mit dem Herzkatheter ist zwar sehr genau, aber teuer, für die Patienten belastend und auch nicht einfach umzusetzen, da sie sich bewegen müssen, während ein Katheter in ihrem Herzen liegt.

Mit der DZHK-Studie haben die Göttinger Wissenschaftler die neue MRT-Untersuchung validiert und bewiesen, dass sie für die Diagnose der HFpEF sehr gut funktioniert und die Katheteruntersuchung so möglicherweise vermieden werden kann. Bevor das Verfahren routinemäßig in der Diagnostik eingesetzt werden kann, sind aber noch größere Studien notwendig. „Bei der FahrradErgometrie Echtzeit MRT handelt es sich um ein komplett neues diagnostisches Verfahren für Patienten mit diastolischer Herzschwäche. Als Nächstes planen wir eine Studie, an der sich mehrere Zentren beteiligen, um zu überprüfen, ob die Methode für die Patienten vorteilhaft ist", so Schuster

Quelle: www.dzhk.de 